\title{
Preparation and Properties of Polylactic Acid/Modified Starch/POE Degradation Materials
}

\author{
Xiaobo Zhang ${ }^{1, *}$ \\ ${ }^{1}$ Yanching Institute of Technology, 065201 Sanhe, China
}

\begin{abstract}
Polylactic acid (PLA) / polyolefin elastomer (POE) / modified starch composites were prepared by melt blending. The composites were characterized by softening point, polarizing microscope, melt flow rate, tensile strength and degradation properties. The results showed that with the increase of modified starch and POE content, phase separation occurred between modified starch and PLA, and the tensile strength decreased compared with pure PLA. Modified starch could increase the crystallinity of PLA to increase the softening point and improve the thermal stability of PLA. When the modified starch and POE content were 15 and 20 parts respectively; the composite material has the best performance. At this time, the softening point was $75^{\circ} \mathrm{C}$ and the tensile strength could reach $47.7 \mathrm{MPa}$. The composite materials in different media showed the quality decreased with the prolongation of degradation time, especially in acidic and alkaline conditions, because the degradation rate of hydrolysis was accelerated.
\end{abstract}

\section{Introduction}

With the progress and development of society, the environmental pollution problems caused by the wide application of plastics are becoming more and more serious. Polylactic acid (PLA), as a kind of material made from starch and biodegradable, has attracted people's attention. The polyester bond in the polylactic acid molecule has the advantages of high modulus and high strength. It can be formed by blow molding, molding and extrusion processing, but its poor impact resistance, heat resistance and hydrophilicity limit its wide application. [1-3]At present, the main method to improve the disadvantages of PLA is to toughen PLA, including copolymerization, blending and processing modification. Blending modification is not only simple, feasible and low cost, but also the blend system can balance the performance of each component, achieving the effect of complementing each other and obtaining a modified material with good comprehensive performance. The commonly used materials for modified PLA are: polybutylene succinate (PBS), poly (adipic acid)/butylene terephthalate (PBAT), poly- $\varepsilon$ caprolactone and so on. [2, 3]

POE has good toughness, good plasticity, excellent dispersion and weather resistance, [4-6] high transparency. Starch has the advantages of a wide range of sources, low cost and short degradation cycle. [7, 8] In this paper, starch and POE were used as toughening modifiers to modify PLA. The effects of various components on the properties of PLA were studied. The application range of PLA was extended.

\section{Experimental}

\subsection{Materials}

Polylactic acid (PLA) (industrial pure, injection molding grade)was purchased from Zhejiang Hai Zheng biological material Limited by Share Ltd; PEO(industrial pure, injection molding grade) was purchased from Changchun Dadi Fine Chemical Co., Ltd.; Modified starch, (analytically pure) was purchased from homemade; Carboxymethyl cellulose(analytically pure) was purchased from Tianjin Yongda Chemical Reagent Co., Ltd.; Cyclodextrin(analytically pure) and Polyvinylpyrrolidone(analytically pure) was purchased from Tianjin Fuchen Chemical Reagent Co., Ltd.; talcum powder(analytically pure) and glycerin(analytically pure) was purchased from Tianjin Dingsheng Chemical Co., Ltd..

\subsection{Experiment process}

\subsubsection{Preparation of modified starch}

The starch is dissolved in water and gelatinized at 85-90 degrees Celsius for 15 minutes. After cooling, acrylic acid, polyvinyl alcohol and initiator are added. The reaction was carried out in a microwave reactor for a certain time, and then silica is added and stirred to obtain a modified starch.

* Corresponding author: zxb_1015@163.com 


\subsubsection{Preparation of composite PLA materials}

The weighted PLA, POE, modified starch and CMC were blended evenly and then add other additives such as glycerin, and then the co-rotating twin screw extruder (SHJ-20, length-diameter ratio 40:1, Nanjing Jeente Electromechanical Co., Ltd.) was used for granulation. The extrusion temperatures are $135^{\circ} \mathrm{C}$ in zone I, $150^{\circ} \mathrm{C}$ in zone II, $170^{\circ} \mathrm{C}$ in zone III, $170^{\circ} \mathrm{C}$ in zone $\mathrm{IV}$ and $135^{\circ} \mathrm{C}$ in zone $\mathrm{V}$ (from feed inlet to outlet). Finally, the obtained pellets were subjected to hot pressing in the range of $150-230^{\circ} \mathrm{C}$ to prepare a PLA modified material.

\subsection{Experimental instrument and test}

Softening Point: According to GB/T 1633-2000 "Determination of softening point temperature of thermoplastic Vicat", the temperature of thermal deformation and Vicat softening point was measured by RHWK-300B type thermal deformation and Vicat softening point tester of Beijing Crown Testing Instrument Co., Ltd.; Polarization microscope test: the sample was melted at $300^{\circ} \mathrm{C}$ for $3 \mathrm{~min}$, pressed into 10 $\mu \mathrm{m}$ thin sheets, and then quenched, the crystal morphology of the material was observed under Chongqing UP203i polarization microscope; Tensile performance test: the sample size is $3 \mathrm{~cm} \times 1 \mathrm{~cm}$, and the mechanical tensile properties are tested by Shenzhen New Sansi E44 electronic universal testing machine (speed $50 \mathrm{~mm} / \mathrm{min}$ ); Degradation performance test: The composite material was prepared into a film by casting method, then the film was placed in the solution, the sampling interval was 5 days, and the weight loss rate of the sample was measured.

\section{Results and discussion}

\subsection{Softening point of composite}

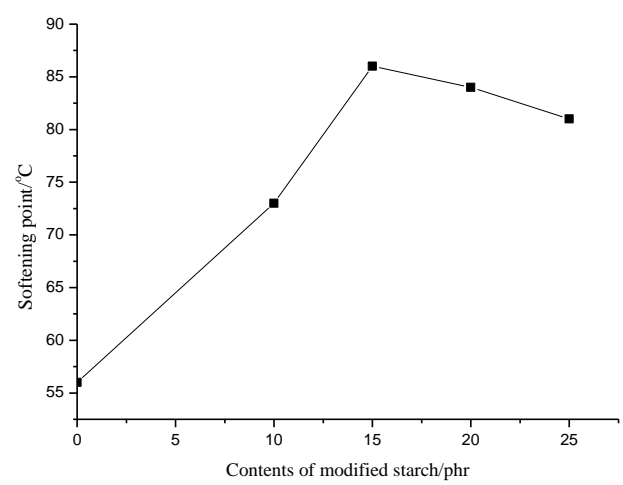

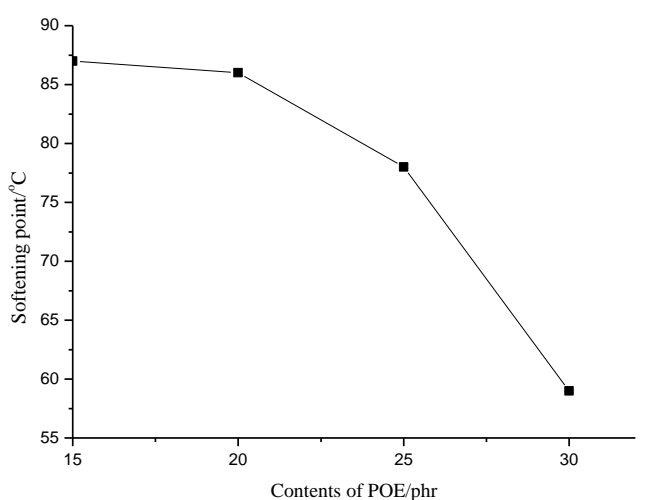

Fig. 1. Different starch and PEO ratios softening point diagram

It can be seen from Fig. 1 that when the quality of the modified starch is increased from 10 parts to 15 parts, the softening point of the blending system increases, but from 15 parts to 20 parts, the softening point temperature begins to decrease. It is known that when the starch is 15 parts, the softening point reaches the best condition of this experiment. With the addition of starch, the crystallinity of PLA was promoted, but after POE is excessive, the heat resistance of the system is poor due to its poor heat resistance. PEO itself plays a toughening effect, therefore its addition leads to The degree of crystallization decreased. When the amount of POE increased to 30 parts, the softening point of the system dropped drastically to 58 degrees. Therefore, in order to improve the heat resistance of the system, the amount of PEO must be strictly controlled.

\subsection{Microstructure of composite materials}

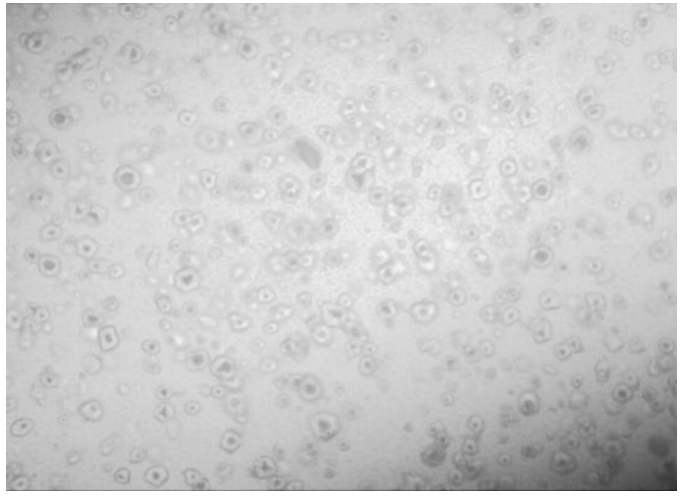

PLA: $\operatorname{starch}=20: 10$ 


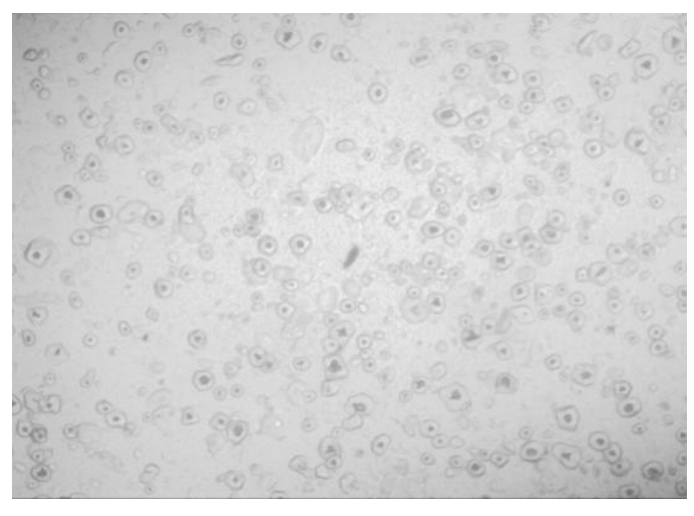

PLA: $\operatorname{starch}=20: 15$

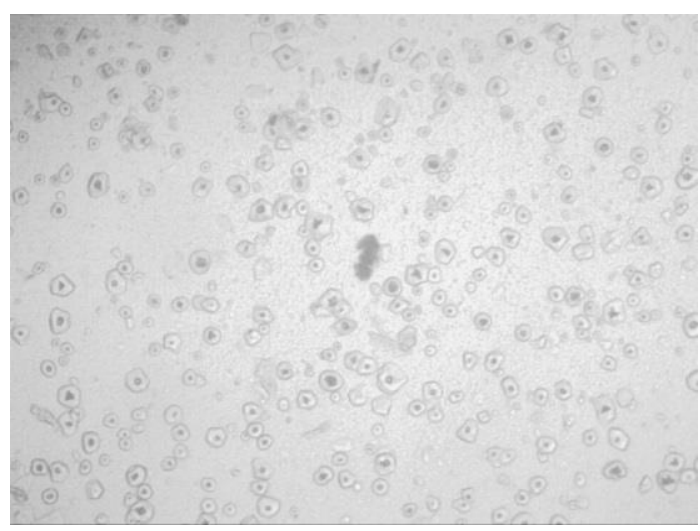

PLA: $\operatorname{starch}=25: 15$

Fig. 2. Polarizing micrographs of starch modified PLA composites with different proportions

Fig. 2 is a polarizing microscope image of the composite material with POE content of 25 parts and different modified starch contents. PLA is a semicrystalline polymer. As can be seen from Fig. 2, there is obvious particle distribution on the surface of the composites, and the PLA is the continuous phase and the starch is the packed phase in the composites. At $140^{\circ} \mathrm{C}$ crystallization conditions, the composite material has crystallized completely, because the interaction force with the matrix PLA restricts the movement of the molecular chain, and forms a rigid interface between them. With the starch content increases, the phenomenon becomes more obvious. As a result, the interfacial compatibility of the composite material is worse, the number of crystals and the size increases. However, the particles are closely distributed and uniformly dispersed, showing higher melt dispersibility with PLA, and the initial and final temperatures of crystallization are much higher than pure PLA. At this time, PLA modified with POE and modified starch has been transformed from the brittle material to the ductile material.

\subsection{Analysis of melt flow rate (MFR) of blend system}
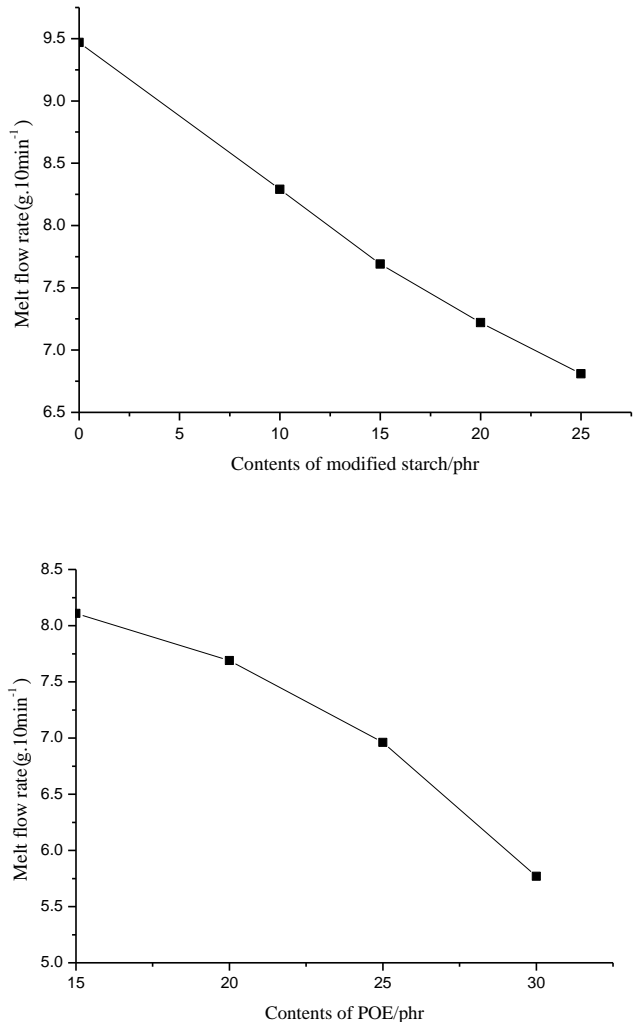

Fig. 3. The melt flow rate of different systems

It can be seen from Fig. 3 that with the increase of POE content in starch and elastomer MFR shows a downward trend, and the effect of POE was greater than that of starch. It shows that the processing performance of the system decreases with the increase of elastomer content. This is because the melt flow rate of the elastomer itself is low. In the blending, the elastomer is dispersed in the PLA melt with tiny droplets. The addition of elastomer increases the internal friction resistance of the blend system, which makes the relative movement of polymer chains difficult and dissipates additional energy, thus reducing the fluidity of the blend system. It is manifested the viscosity increased and the MFR value decreased. Therefore, the proper proportion should be chosen to facilitate the processing of the blends. 


\subsection{Mechanical properties of composite materials}
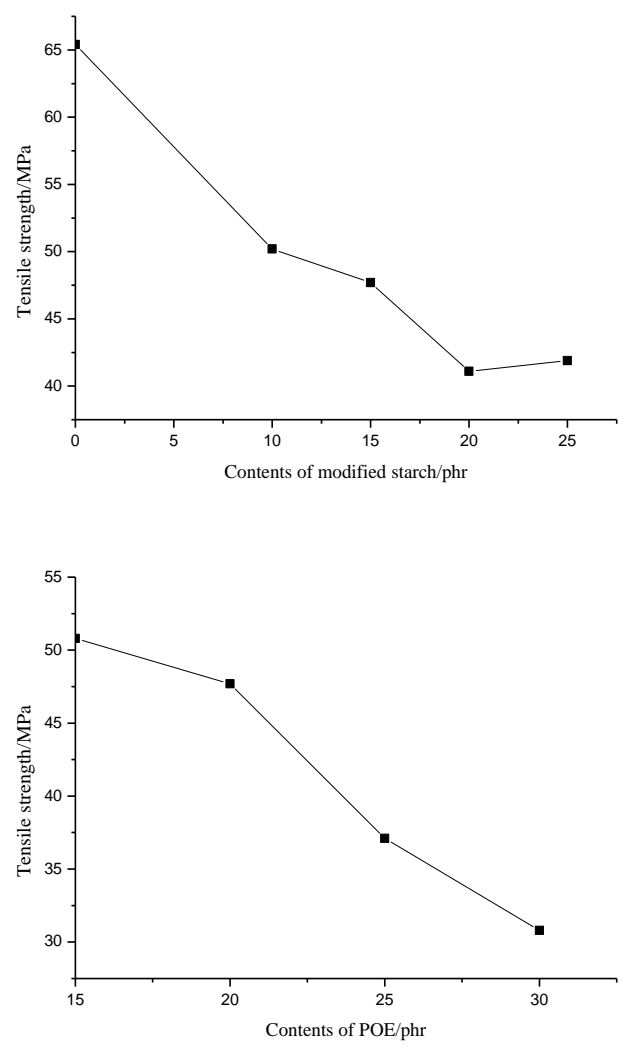

Fig. 4. The mechanical properties of PLA and PLA blends

Fig. 4 shows the mechanical properties of PLA blend with different contents of modified starch. The tensile strength of pure PLA is $65.4 \mathrm{MPa}$. The tensile strength of the composites decreased first and then increased with the increase of modified starch content. When the starch content is small, the tensile strength of the material is mainly derived from polylactic acid. But because both PLA and starch are brittle materials, phase separation occurs between the modified starch and PLA, the interfacial adhesion is deteriorated, and the tensile strength decreases. With the increase of starch content, glycerol makes PLA-COOH and starch-OH cross-linked excessively, restricting the movement of the interface between PLA and starch, thus increasing the small molecule chain forging of PLA, accelerating the crystallization and improving the crystallinity, so the tensile strength of PLA composites increases.

With the increase of POE content, the tensile strength remains basically unchanged at first and then decreases. At higher POE content, the tensile strength remains at 30 $\mathrm{MPa}$. The addition of POE elastomers has a toughening effect on PLA blends. The addition of POE elastomer weakened the interaction force between PLA macromolecules. However, due to the low rigidity of POE itself, when the amount of POE was too much, the POE forming the elastic toughening phase microspheres in the PLA matrix increases the POE phase microspheres with the increase in content, which can not further strengthen the toughness, and the tensile strength decreases significantly.

\subsection{Degradation properties of composite materials}

The degradation performance of the composites is shown in Fig. 5. It can be seen from Fig. 5 that in the acid, alkali and neutral medium, the quality of the composite material tends to decrease with the extension of time. The modified polylactic acid can be degraded in acid, alkali and neutral medium, and its degradation rate is slowed down in turn, especially in alkaline media. This is because the side chain of polylactic acid can be subjected to deesterification in alkaline, which is more easily hydrolyzed than under acidic conditions. Moreover, the modified starch has certain hydrophilicity, and its addition can form a phase-separated blend with PLA, which can accelerate the hydrolysis of the sample.

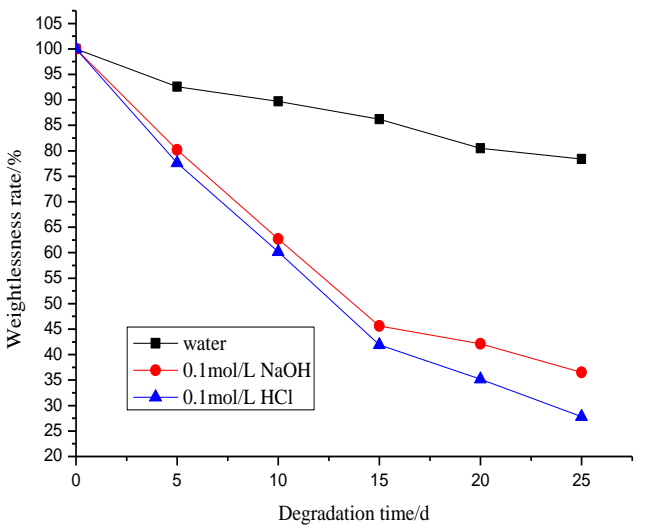

Fig. 5. Degradation curve of composite materials in different medium

\section{Conclusions}

The composite materials of modified starch and POE modified PLA were prepared by melt blending method. Modified starch and POE can improve the crystallization of PLA to some extent. Modified starch, POE and PLA can be effectively bonded, which improves the tensile strength and heat resistance of the composite, but the flow performance decline slightly. When the content of modified starch and POE is increased at 15 and 20 parts respectively, the tensile strength can reach $47.7 \mathrm{MPa}$ and softening point is $75^{\circ} \mathrm{C}$. The weight loss of the composite materials in different media is obviously different, and the degradation rate in alkaline condition is much faster than that in acidic and neutral conditions.

\section{References}

1. K. W. Kim, S. I. Woo, Macromol Chem Phys. 203,2245-2250(2002)

2. K. Das, D. Ray, I. Banerjee, J Appl Polym Sci. 118,143-151(2010) 
3. H. L. Wang, M. G. Sheng, T. J. Shi, Polym. Mater.Sci. \& Eng. 20(6), 20-23(2004)

4. L. Fang, J. Li, R.R. Qi, Engineering Plastics Application., 37(6),5-8(2009) (In Chinese)

5. F. Du, China plastics. 25(11),39-41(2011) (In Chinese)

6. H. T .Oyama, Polymer. 50, 745-751 (2009)

7. D. Garlotta, W. Doane, R. Shagren, J.Appl.Polym.Sci. 88(7), 1775-1786(2003)

8. O. Martin, L. Averous, Polymer. 42(14), 62096219(2001) 